\title{
3D DIC tests of mirrors for the single-mirror small-size telescope of CTA
}

\author{
M. Rataj $^{1}$ - M. Malesa ${ }^{2}$ - M. Kujawińska ${ }^{2}$.

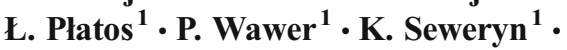 \\ K. Malowany ${ }^{2}$
}

Received: 22 December 2014 / Accepted: 7 April 2015 /Published online: 9 June 2015

C The Author(s) 2015. This article is published with open access at Springerlink.com

\begin{abstract}
The Cherenkov Telescope Array (CTA) is the next generation very high energy gamma-ray observatory. Three classes of telescopes, of large, medium and small sizes are designed and developed for the observatory. The single-mirror option for the small-size telescopes (SST-1M), of $4 \mathrm{~m}$ diameter, dedicated to the observations of the highest energy gamma-rays above several $\mathrm{TeV}$, consists of 18 hexagonal mirror facets of $78 \mathrm{~cm}$ flat-to-flat. The goal of the work described in this paper is the investigation of a surface shape quality of the mirror facets of the SST-1M CTA telescope. The mirrors measured are made of composite materials formed using sheet moulding compound (SMC) technology. This solution is being developed as an alternative to glass mirrors, to minimize the production cost of hundreds of mirrors for the network of telescopes, while retaining the optical quality of the telescope. To evaluate the progress of design, production technology and the mirrors' functionality in operating conditions, the threedimensional (3D) Digital Image Correlation (DIC) method was selected and implemented for testing selected mirrors. The method and measurement procedure are described. The novel measurement approach based on 3D DIC has been proven to be well suited to the investigation of the mirrors' behavior with temperature, producing the necessary accuracy.
\end{abstract}

Keywords Telescope mirrors · SMC technology · Composite mirror - Optical metrology Digital image correlation method

M. Rataj

rataj@cbk.waw.pl

1 Space Research Centre of the Polish Academy of Sciences, Bartycka St. 18A, 00-716 Warsaw, Poland

2 Institute of Micromechanics and Photonics, Warsaw University of Technology, Św. A. Boboli St. 8, 02-525 Warsaw, Poland 


\section{Introduction}

The Cherenkov Telescope Array (CTA) project is an initiative to build the next generation ground-based very high energy gamma-ray instrument [1]. Gamma rays with photon energies ranging from several tens of $\mathrm{GeV}$ to beyond 100 $\mathrm{TeV}$ will be detected by ground-based telescopes that observe the Cherenkov light emitted in air showers created by gamma rays. To achieve its goals, the array will consist of at least three types of telescopes. The highest energy photons will be observed by Small Size Telescopes (SSTs). About 70 of these will be located in the southern hemisphere array.

A consortium of Polish, Swiss and German institutions is developing an SST prototype called the Single Mirror Small Size Telescope (SST-1M) [2]. The telescope is based on a Davies-Cotton design [3] proven in existing Imaging Atmospheric Cherenkov Telescopes (IACTs). The novel element of the telescope will be a fully digital and lightweight camera based on Geiger avalanche photodiodes [4]. The telescope has a focal length of $5.6 \mathrm{~m}$ and offers $9^{\circ}$ field of view. The structure of the telescope is formed by a tower, a dish support structure, a counterweight and a camera mast. 18 mirror facets form the telescope dish of $4 \mathrm{~m}$ diameter. The mirror tiles are hexagonal, $0.78 \mathrm{~m}$ flatto-flat in size. The effective light collecting area, corrected for the camera and mast shadowing, is $6.47 \mathrm{~m}^{2}$. Each mirror will be attached to the dish support structure using a set of actuators allowing for the mirror alignment.

In the design phase different solutions and materials are considered for CTA mirrors [5]. Glass mirrors are the baseline solution for the SST-1M. However, in parallel, composite mirrors are being developed by means of sheet moulding compound (SMC) formation [6] to provide a lightweight, reliable and costeffective alternative. SMC is a proven technology in the automotive industry and some of its advantages include: only a one-step process is needed to produce the substrate, it is a fast process which takes $3 \mathrm{~min}$ only and the material shows no shrinkage. In addition, the top surface of the composite mirror does not require polishing, as a smooth surface is obtained. This solution is being investigated to minimize production cost of hundreds of mirrors for the network of telescopes, whilst simultaneously fulfilling the telescope's optical requirements. Ensuring that the surface shape of the mirror facets is maintained under all operation conditions is an essential requirement for the performance of the telescope.

Therefore investigations of the thermal behavior of composite mirrors manufactured using different designs and manufacturing techniques are crucial for optimizing both the design and the manufacturing processes. The measurement method should provide such quantities as shape, deformation and strain distributions as a function of the temperature. The three dimensional Digital Image Correlation (3D DIC) method [7] was selected as the best fitted full-field, vision-based measurement method.

In Section 2 a description of the SST-1M composite mirror and its basic tests are presented. In the next two sections a short description of the 3D DIC method is provided and the measurement system and methodology are described. In Section 5 the results of example measurements of the mirrors are presented in detail and the usefulness of this testing method is assessed. 


\section{SST-1M composite mirror}

The composite mirrors are being developed as an alternative to glass mirrors. The solution is based on a composite substrate formed using SMC technology. Its major feature is that the mirror substrate is composed of one isotropic, thermally conductive material and there is no glass in the structure. The top surface of the composite does not require polishing, and its smooth surface is obtained through the use of a high gloss mold. Since no glass is used as a front surface of the substrate, the coating includes not only reflective and protective layers but also an intermediate layer (lacquer or gel-coat) applied in order to reduce the surface roughness of the composite substrate, and improve both the mechanical resistance and coating adhesion. The achieved surface roughness $\left(\mathrm{R}_{\mathrm{a}}\right)$ is below $5 \mathrm{~nm}$, which is considered adequate for this application. The reflectivity of the mirror surface is obtained by the use of the intermediate layer followed by aluminium coating and $\mathrm{SiO}_{2}$ protective layer. One of the first prototypes, of $0.4 \mathrm{~m}$ flat-to-flat, is shown in Fig. 1.

At the presented stage of development of this technology, the surface quality of mirrors made of composite materials does not reach the quality level of glass and ceramic mirrors. Fortunately the mirrors of the SST-1M do not have very demanding optical the Point Spread Function (PSF) requirements, these being that $85 \%$ of the energy should fall within a circle of $32 \mathrm{~mm}$ diameter and $80 \%$ of that energy within a $17 \mathrm{~mm}$ diameter circle, over a wavelength range of 300 to $550 \mathrm{~nm}$. However this optical performance must be sustained over the whole temperature operating range from -15 to $25^{\circ} \mathrm{C}$. For testing, the temperature range was increased to $40^{\circ} \mathrm{C}$, to have a picture of the temperature behavior of the mirror in non-operating temperature range. The optical requirements, presented above, are for the first version of the SST telescope. Next version of the telescope has a proportionally reduced focal length and dimensions of the detector. Results of the presented analysis are easy to transfer to the new version by scaling. But the key point for our activities was to evaluate the progress of development of the applied technology.

The optical and geometrical parameters of the prototype mirrors have been measured by means of a Form Talysurf PGI 830 profilometer (surface roughness), a coordinate measuring machine and a 2-f optical measurement system. In the 2-f measurement system, a point-like light source (a pigtailed diode laser with $\lambda=405 \mathrm{~nm}$ or $635 \mathrm{~nm}$ ) is placed at the distance which is equal to the mirror radius of curvature (equivalent to twice the focal length). A detector is placed at the same distance to register the image
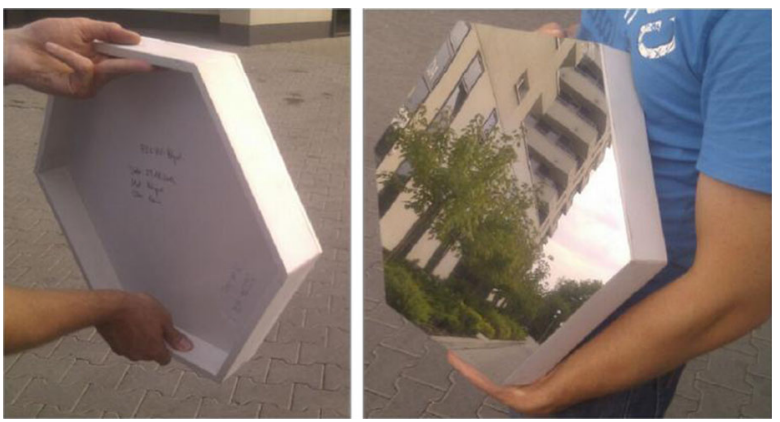

Fig. 1 The composite mirror prototype - rear and front side 
(spot) formed by laser beam reflected by the mirror under test. In this configuration we are able to measure the PSF shape and dimensions, the radius of curvature and the reflectance of the mirror into the focal spot under laboratory conditions. All these parameters are dependent on the shape of the mirror surface. This simple measurement method based on a 2-f system unfortunately cannot be used for the mirror tests inside a climate chamber. The allowed change in the radius of curvature of the mirror over a $55{ }^{\circ} \mathrm{C}$ temperature range is $\pm 2 \mathrm{~m}$, which results in a surface deformation of $\pm 20 \mu \mathrm{m}$. This value had encouraged us to use a simple image processing based method for shape and deformation measurements, namely the 3D Digital Image Correlation Method (DIC). The additional motivation to choose 3D DIC as the experimental method is its capability to determine strains in an mirror exposed to load (including thermal load), which must be considered for the SST-1M.

\section{Three dimensional (3D) digital image correlation method}

DIC is a non-coherent light based method which enables both full-field measurements of shape and displacements and the determination of strains in a wide variety of objects $[7,8]$. The two-dimensional version of the DIC (2D DIC) measurement procedure is very simple, and requires the capture of a series of images of a tested object before and after load (or during loading). The surface of the measured object needs to exhibit a random texture (a speckle pattern). In most cases it is necessary to cover the measured object with such a pattern before the measurements start. One of the images in a series is selected as a reference image for all subsequent analyses. The reference image is divided into small rectangular regions (subsets) consisting of $\mathrm{N} \times \mathrm{N}$ pixels. The dimensions of the subsets are dependent on the quality and the size of the random pattern on the measured object. The DIC algorithm then tracks the position of each subset relative to the reference image in all other images of the series. Corresponding subsets are matched by finding the maximum of the zero-normalized cross-correlation function coefficient (or any other correlation metric). The difference in position between a reference subset and its matched equivalent defines the in-plane displacement vector at the subset's origin ( $U$ denotes displacements along ' $x$ ' axis and $V$ denotes displacements along ' $y$ ' axis). Repeating this process over the area of interest yields a displacement map, that is sampled at an interval defined by the subset size and the separation of subsequent subsets selected for analysis (step size). Sub-pixel accuracy is achieved by sophisticated interpolation methods.

In 3D DIC, a pair of images is acquired simultaneously by two cameras viewing the object from different directions. DIC, combined with stereo-vision and triangulation methods [9], provides maps of the shape together with out-ofplane and in-plane displacements of an object which have occurred between acquisitions of images. The 3D DIC measurement procedure is presented in Fig. 2. The cross-correlation function is calculated twice: for the first time in order to determine $(\mathrm{x}, \mathrm{y}, \mathrm{z})$ coordinates of each subset in a stereo-image pair and for the second time in order to match all subsets against corresponding subsets in other images of the measurement series (providing in-plane and out-of-plane displacements). In the Figures hereafter, displacements along $\mathrm{x}$ and $\mathrm{y}$ axes are denoted as $U$ and $V$, while displacements along $\mathrm{z}$ axis are denoted as $W$. 


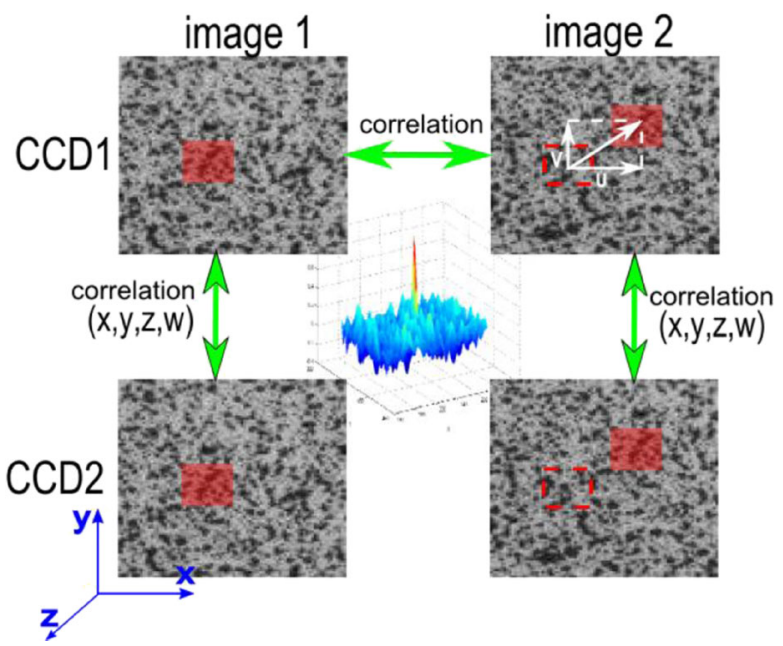

Fig. 2 Scheme of 3D DIC measurement procedure. An example subset from the reference image (the red rectangle in the middle) is matched against similar subsets in other images of the series; the contour plot in the middle is an example distribution of the correlation coefficient- the peak indicates the best matched position of the subset

The sensitivity of 2D DIC depends on the dimensions of the fields of view and the resolution of the cameras. In the $3 \mathrm{D}$ DIC method, the sensitivity also depends on the triangulation angle between the cameras.

Using the DIC method in experimental tests provides much more data than pointwise techniques. The measurements are carried out using thousands or millions of points on the measured object simultaneously and in all directions. The data obtained can be easily post-processed in order to calculate deformations or strain maps. The feasibility of 3D DIC and its modifications in different domains have been proven in a number of scientific articles published in recent years [10-18].

\section{Measurement setup and methodology}

The mirror was specially modified before the measurements by spraying with white paint and later introducing a speckle (random) pattern with black paint. The 3D DIC measurement system consisted of two AVT Pike $16 \mathrm{Mpx}$ cameras equipped with $28 \mathrm{~mm}$ focal length lenses. The measurement setup is presented in Fig. 5b. According to [7] the accuracy of displacement measurements with these cameras and over a $0.6 \mathrm{~m} \times 0.6 \mathrm{~m}$ FOV would be as good as $2 \mu \mathrm{m}$ (corresponding to $0.01 \mathrm{px}$ accuracy). Due to experimental errors $[19,20]$ and observation through the window, the estimated accuracy was approximately $10 \mu \mathrm{m}$.

In each series of measurements, the mirror was placed in a FEUTON K-400 climate chamber, which provided the temperature control. In order to ensure the optimal illumination conditions, the chamber was covered inside with black cardboard (to reduce the influence of light reflections) and the LED light was placed inside the chamber. The 3D DIC cameras monitored the mirror through a window in the door of the chamber. The window consisted of 5 glass layers, which influenced the accuracy of 
the measurements. In order to estimate the systematic error caused by the observation window two shape measurements of the mirror at room temperature were performed: the first with the doors of the climate chamber open, and the second with closed doores. The results are presented in Fig. 3.

The measurements through the window introduced a modulation of $\mathrm{z}$ coordinate with an amplitude of approximately $0.01 \mathrm{~mm}$, which is small when compared to the recorded $W$ displacements, which are of an order of $0.1 \mathrm{~mm}$.

Figure 4 shows the temperature cycle implemented during the tests in the climate chamber, together with the indication of the time points when the sequential measurements were taken.

In order to ensure a stable temperature and homogeneous temperature distribution, the mirror was kept at each test temperature for about one hour before performing the measurement. The temperature of the mirror was measured with a set of point-like sensors, glued on the rear of the mirror (Fig. 5a). Cooling or heating the mirror by $20{ }^{\circ} \mathrm{C}$ took approximately $10 \mathrm{~min}$.

Images of the measured mirror were acquired at $60 \mathrm{~s}$ intervals, which resulted in 400 pairs of frames captured by the DIC cameras. The measurement setup is presented in Fig. $5 b$.

The 3D DIC analysis presented below was made with the CCI software, developed at the Institute of Micromechanics and Photonics, Warsaw University of Technology, in collaboration with KSM Vision (http://ksmvision.pl/en 2014) and with VIC 3D software developed by Correlated Solutions (www.correlatedsolutions.com 2014). All $U, V$ and $W$ displacement maps (or values) presented below correspond to the $\mathrm{x}, \mathrm{y}$ and $\mathrm{z}$ axes respectively. Coordinate systems are overlaid on the displacement maps.

\section{Measurement results}

This section presents the detailed results of our sample measurements. At the first experimental stage the complete mirror structure (the substrate with intermediate layer) was examined in the climate chamber over the full range of temperatures following the scheme described in Fig. 4. Reference frames were taken at $20^{\circ} \mathrm{C}$. The measurements were then repeated on a mirror having only the substrate and no intermediate layer. The main goal in these two series of measurements was to evaluate the possible influence of
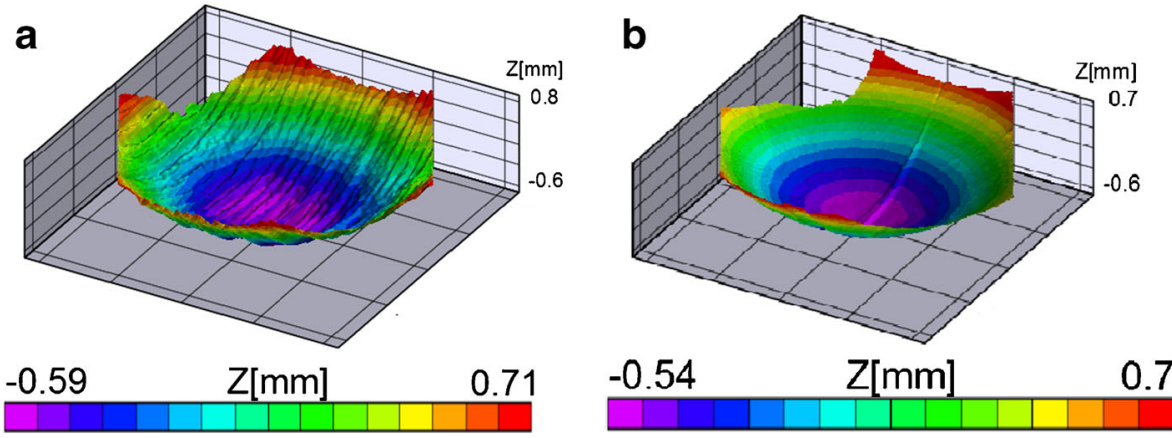

Fig. 3 Point cloud of the measured object acquired with 3D DIC method: (a) observation through the window, (b) direct observation (with opened door) 


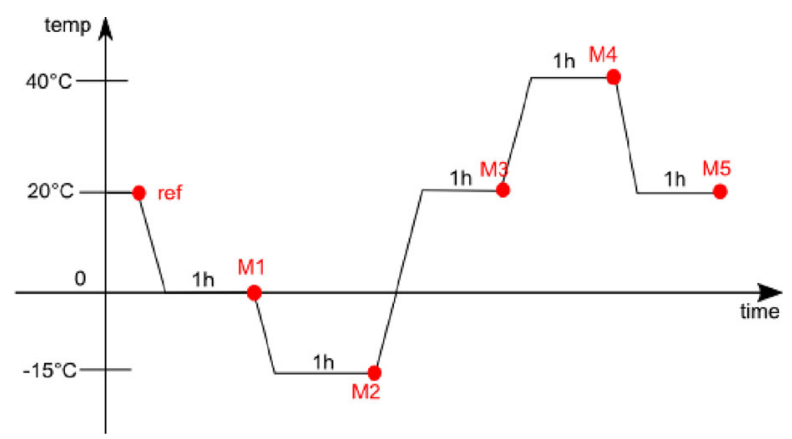

Fig. 4 Cycle of temperature changes implemented during the measurements; steady states are indicated with red dots

the intermediate layer on the mirror deformation when the ambient temperature is changed.

Example $U, V$ and $W$ displacement maps (obtained after rigid body movements have been removed) of the mirror with the intermediate layer are presented in Fig. 6.

As one can observe in Fig. 6, the mirror's radius of curvature decreased with decreasing temperature (negative $W$ displacements in the centre of the mirror and positive $W$ displacement at the edges) and increased with increasing temperature (positive $W$ displacements in the centre of the mirror and negative $W$ displacement at the edges). It is also clear that the mirror returns to its original shape once it returns to its initial temperature of $20^{\circ} \mathrm{C}$. However comparing the maps presented in Fig. $6 \mathrm{c}$ and e, which theoretically were captured at the same temperature, some differences can be observed. There are a few reasons of these differences. At first it should be noted that at $20{ }^{\circ} \mathrm{C}$ the displacement values are smaller by one order of magnitude when compared to other states of load. Actually, due to the systematic error caused by the observation through window of the chamber (see Fig. 3), the values obtained in states M3 and M5 are close to the accuracy limit of the method. The influence of the observation through the window is reflected by the high frequency variations of the presented displacement maps. Secondly, the temperature has been measured with approximately $0.5^{\circ} \mathrm{C}$ uncertainty and additionally the temperature could have not been distributed homogeneously over whole surface of the mirror (pointwise sensors measurements differs from each
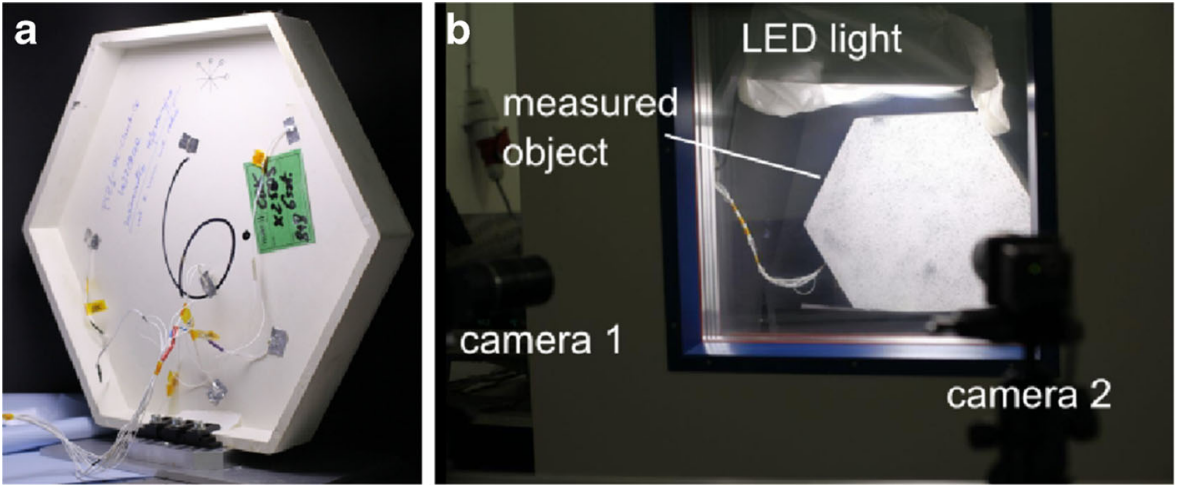

Fig. 5 (a) Pointwise temperature sensors glued at the measured mirror, (b) 3D DIC measurement setup: cameras of the system and measured mirror enclosed in the climate chamber 


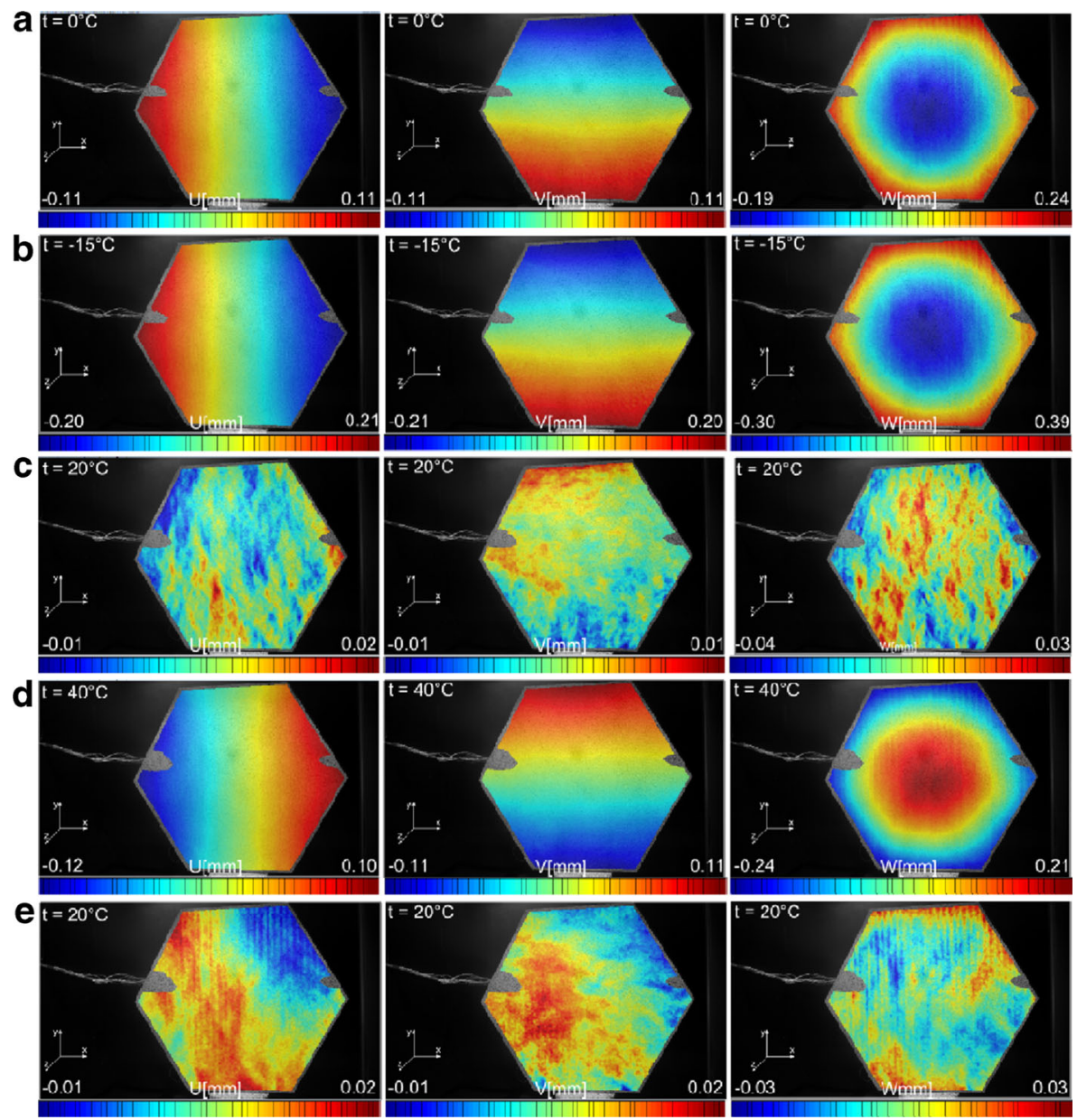

Fig. $6 U, V$ and $W$ displacement maps in steady states of the mirror: (a) $\mathrm{M} 1,0{ }^{\circ} \mathrm{C},(\mathbf{b}) \mathrm{M} 2,-20{ }^{\circ} \mathrm{C},(\mathbf{c}) \mathrm{M} 3$, $20{ }^{\circ} \mathrm{C}$, (d) $\mathrm{M} 4,40{ }^{\circ} \mathrm{C}$, (e) $\mathrm{M} 5,20{ }^{\circ} \mathrm{C}$. The maps are at different colour scales, tailored to the minimum and maximum values found in each map

other by a maximum of $0.5^{\circ} \mathrm{C}$ ). All of these facts influenced the displacement maps acquired in steady states M3 and M5 (even though theoretically temperature was $20^{\circ} \mathrm{C}$ in both states). In order to show a more detailed analysis, the displacements extracted from selected points and along selected lines have been plotted as a function of time (actually as a function of file number). The locations of points P1-P7 and line L2 are presented in Fig. 7. The same data processing procedure has been applied to measurements with and without the intermediate layer.

Displacements $U, V$ and $W$ extracted from points P1-P7 are presented in Fig. 8 together with the temperature changes (measured in the centre of the mirror). Plots in Fig. $8 \mathrm{a}, \mathrm{c}$ and e represent the first series (for the mirror with the intermediate layer) and plots in Fig. 8 b, $d$ and $\mathrm{f}$ represent the results of the second measurement series (the mirror without intermediate layer). In order to minimize the influence of $\mathrm{z}$ coordinate modulations (see Fig. 3), the results at each point have been averaged over a small circle (radius of about 10 pixels). 


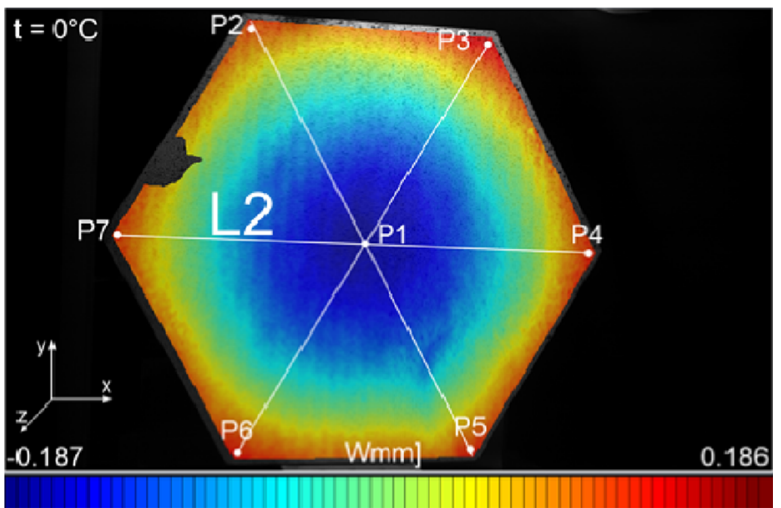

Fig. 7 Example $W$ displacement map with overlaid points and lines of further analysis

One can observe in Fig. 8 that the temperature of the mirror did not stabilize at negative temperatures. For example, at a steady climate chamber temperature of $-15{ }^{\circ} \mathrm{C}$ (M2) the temperature measured on the mirror was $-18{ }^{\circ} \mathrm{C}$, whereas this was not the case at temperatures above $0{ }^{\circ} \mathrm{C}$. It can also be observed that
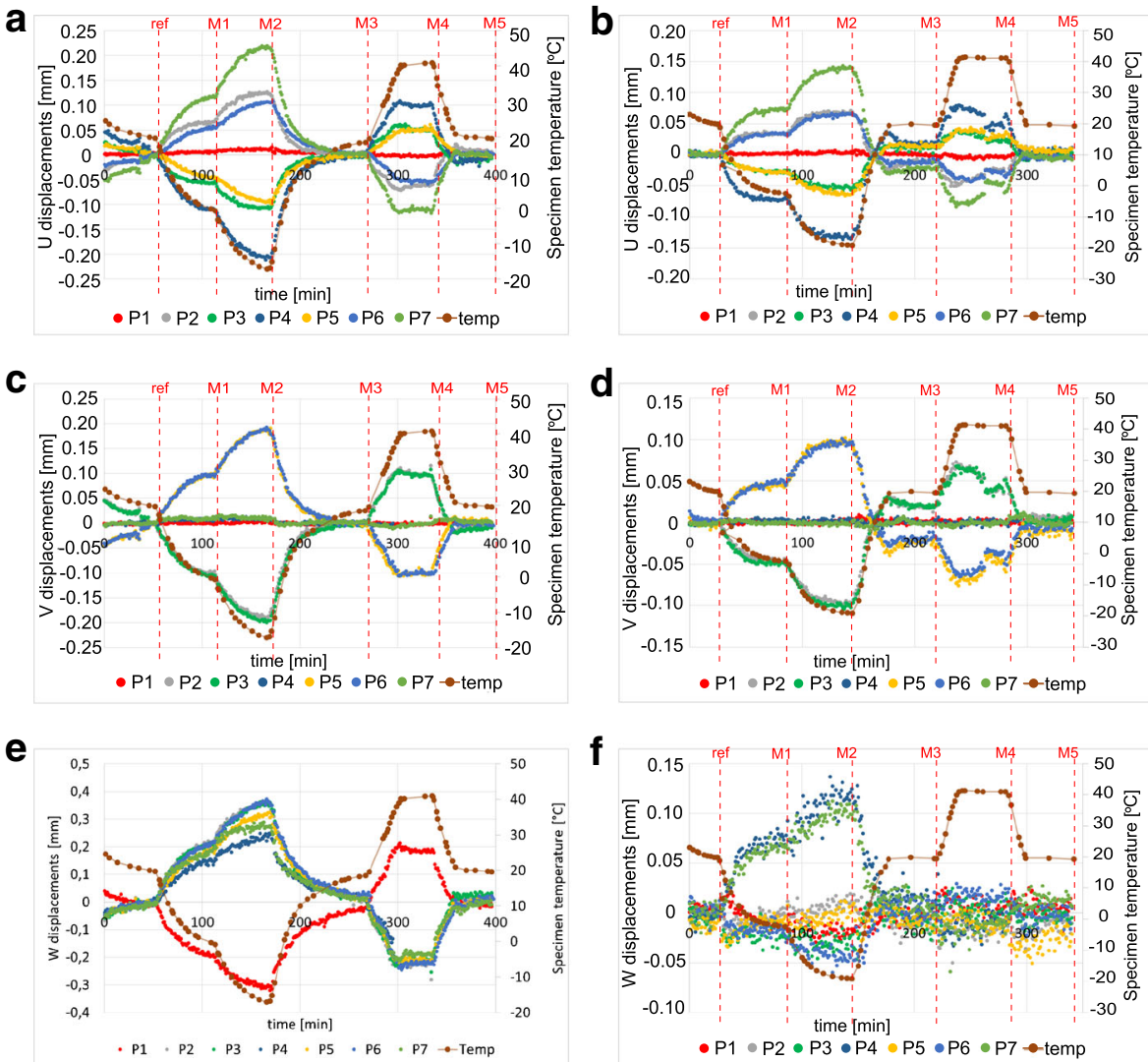

Fig. 8 Displacements extracted from points P1-P7: the first series displacements (a) $U$, (c) $V$, (e) $W$ and the second series displacements (b) $U$, (d) $V$, (f) $W$ 
the specimens shrink as a result of the temperature variations. In-plane displacements $U$ and $V$ at points P2-P7 are distributed symmetrically, while at point P1 their values are negligibly small. All components of displacement vector return to 0 when the temperature returns to the initial value of $20{ }^{\circ} \mathrm{C}$ (M3 and M5). This observation is confirmed in the displacement maps (Fig. 6) as well as in the displacements plots (Fig. 8). The very small displacement values that were detected in the steady states M3 and M5 could be an effect of very small temperature differences between the initial and tested states or could be introduced by performing the measurement through the window (vertical displacement gradients are clearly seen on maps with small displacement values). The results of $U$ and $V$ displacement measurements were similar in both series (Fig. 8 a and b). Slightly bigger maximum values were obtained in measurements of the mirror without the intermediate layer, but in both cases displacements are close to zero at the initial temperature of $20{ }^{\circ} \mathrm{C}$. It is different in the case of out-of-plane displacements. The biggest out-of-plane displacements were detected at point P1 (in the centre of the mirror). $W$ displacements at other points had the opposite sign to displacements in P1. The trend in the $W$ displacements indicates the change of the mirror's radius induced by the changing temperature. Much bigger $W$ displacements were obtained in measurements of the complete mirror (Fig. 8e) when compared to the measurements of the mirror without intermediate layer (Fig. 8f). This indicates the considerable influence of the intermediate layer on the overall sensitivity of the mirrors to ambient temperature variations.

In further analysis, trends of the deformations of the mirrors were evaluated. The plots illustrating displacements $U, V$ and $W$ extracted from points along the line L2 as a function of time are presented in Fig. 9, for the mirror with an intermediate layer.

In Fig. 9 one can observe a symmetrical trend of deformations of the measured mirror. Zero displacements did not occur in the centre of the mirror, indicating the deformation of the spherical shape of the mirror which can be a source of additional aberrations. This effect could be caused by the influence of the thickness of the side walls of the mirror.

The changes of $U$ and $V$ displacements in time (Fig. 9a and b) and in space (Fig. 6) caused significant strains in the element. The variations of the strains in time $\left(\varepsilon_{\mathrm{xx}}, \varepsilon_{\mathrm{yy}}\right)$ calculated along the line L2 are presented in Fig. 10.

The strains $\varepsilon_{\mathrm{xx}}$ and $\varepsilon_{\mathrm{yy}}$ at $20{ }^{\circ} \mathrm{C}$ (ref, M3, M5) have very small values, while they increase to significant positive values (tensile strains) at $40{ }^{\circ} \mathrm{C}$ (M4) and decrease to comparable negative values (compressing strains) at $-15{ }^{\circ} \mathrm{C}$ (M2). This behavior suggests that significant fatigue stresses will occur in the mirrors at normal operating temperatures. This is an undesirable feature which has to be avoided.

In the last step of the data analysis, the radii of curvature of the mirrors have been calculated. This has been done by fitting a sphere using a least squares method to the point clouds ( $\mathrm{x}, \mathrm{y}, \mathrm{z}$ coordinates) of the mirrors acquired in consecutive steady states. The comparison between radii of curvature of mirrors with and without an intermediate layer are presented in Table 1. The values of calculated radii are given together with the uncertainty calculated as a standard deviation of residuals of all points from the fitted point cloud. Differences of 

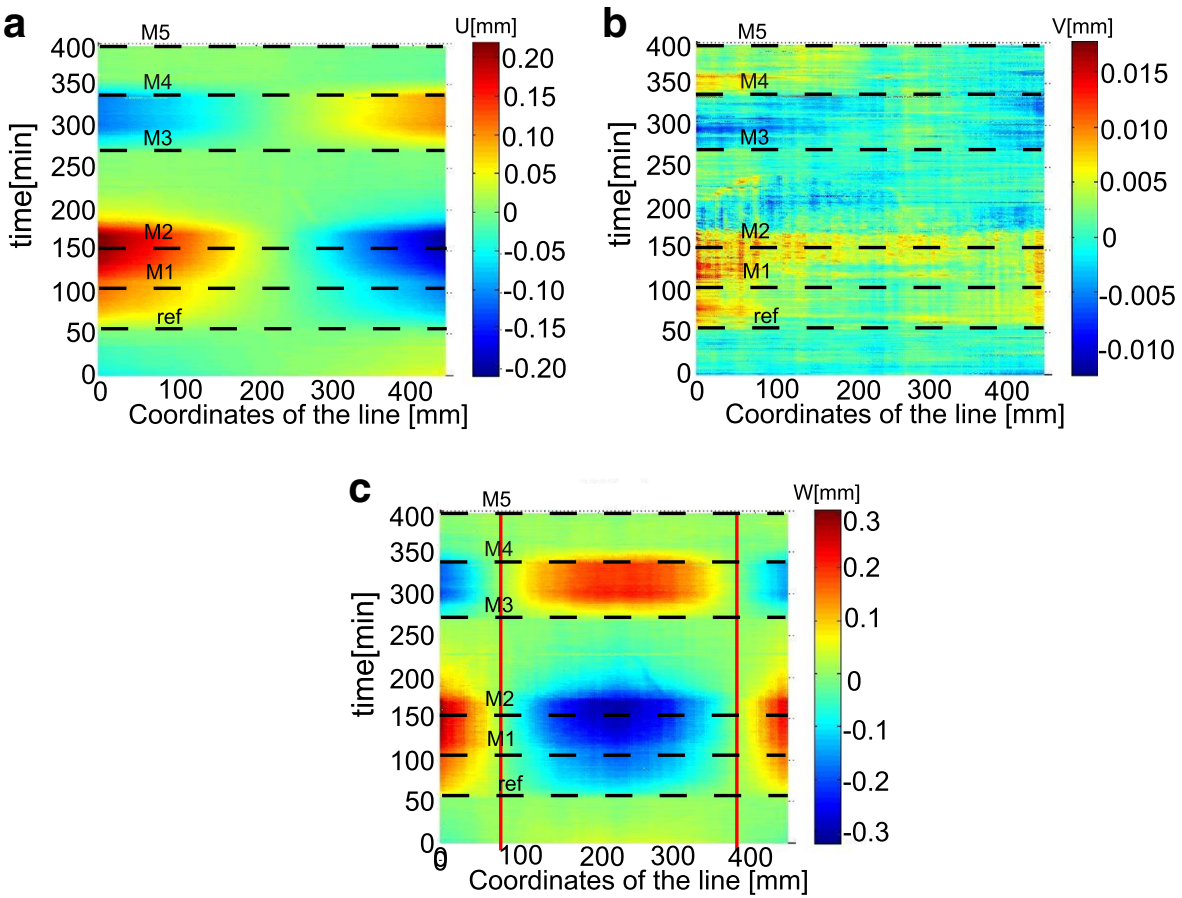

Fig. 9 Displacements extracted from points along line L2 as a function of time: (a) U displacements, (b) V displacements and (c) $W$ displacements. The maps are presented at different colour scales, tailored to the minimum and maximum values found in each map

radii obtained at approximately the same temperature are caused by both uncertainties of temperature measurement and sphere fitting procedure.

At the initial temperature $20{ }^{\circ} \mathrm{C}$ (ref), the radii of curvature of both mirrors were approximately $15.5 \mathrm{~m}$. Also in the steady states M3 and M5 (when the temperature was $20{ }^{\circ} \mathrm{C}$ again) the radii of curvature returned to their initial values. However, the difference between the radii of the mirrors with and
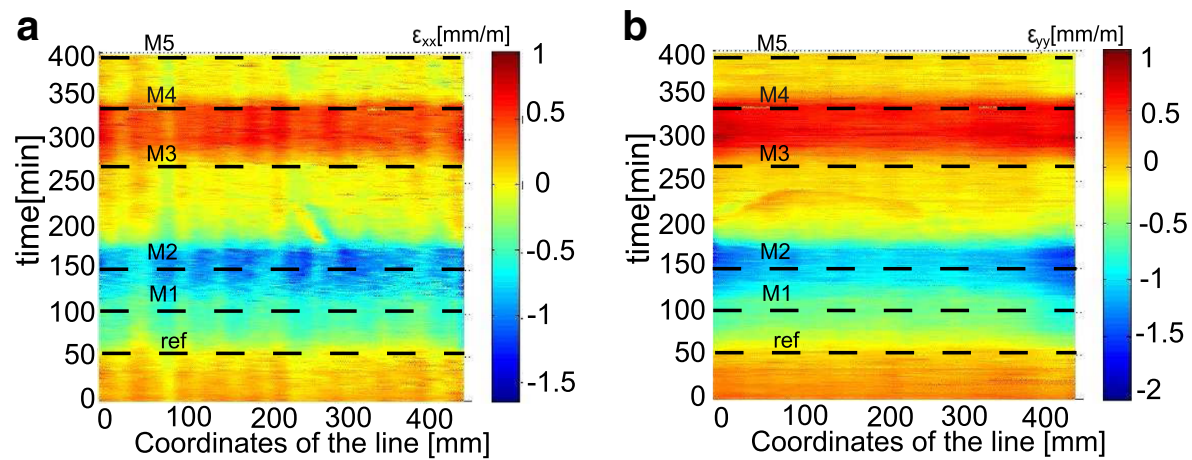

Fig. 10 Strains calculated along line L2 as a function of time: (a) strains $\varepsilon_{\mathrm{xx}}$, (b) strains $\varepsilon_{\mathrm{yyy}}$. The maps are at different colour scales, tailored to the minimum and maximum values found in each map 
Table 1 Variation of the radii of curvature of the SMC mirrors with temperature

\begin{tabular}{lrll}
\hline Steady state & Temperature $\left[{ }^{\circ} \mathrm{C}\right]$ & \multicolumn{2}{l}{ Radius $[\mathrm{m}]$} \\
\cline { 3 - 3 } & & With intermediate layer & Without intermediate layer \\
\hline ref & $20 \pm 0.5$ & $15.43 \pm 0.09$ & $15.53 \pm 0.17$ \\
M1 & $0 \pm 0.5$ & $12.40 \pm 0.09$ & $15.35 \pm 0.17$ \\
M2 & $-15 \pm 0.5$ & $11.03 \pm 0.09$ & $15.07 \pm 0.17$ \\
M3 & $20 \pm 0.5$ & $15.59 \pm 0.10$ & $15.55 \pm 0.17$ \\
M4 & $40 \pm 0.5$ & $20.81 \pm 0.10$ & $15.59 \pm 0.17$ \\
M5 & $20 \pm 0.5$ & $15.39 \pm 0.09$ & $15.48 \pm 0.17$ \\
\hline
\end{tabular}

without the intermediate layer can be clearly observed in the steady states M2 and M4. The radius of curvature of the mirror without the intermediate layer remained the same within errors throughout the measurements, while the variations of the radius of curvature of the mirror with intermediate layer changed significantly with temperature. The main cause of this phenomenon is the difference in the coefficient of thermal expansion of the substrate and the intermediate layer. The intermediate layer thickness was initially assumed to be small such that the influence on thermal deformations is negligible. However, in practice, for technical reasons, this layer has a thickness of several $\mathrm{mm}$, which causes significant variation in the radius of curvature of the structure. To minimize this negative impact, it is proposed to make the structure symmetrical by applying an intermediate layer on both sides of the substrate. This technology update is currently under development.

\section{Conclusion}

The proposed method of measurement of the surface deformation and accompanying strains has proven to be particularly useful for the mirror tests in the operating temperature range. The measurement accuracy obtained, which was better than $10 \mu \mathrm{m}$, was sufficient to estimate the deformation of the surface during tests in the thermal chamber. The random texture on the mirrors (a speckle pattern) produced by paint is easy to remove without any damage to the surface. The results obtained helped to identify the main (technological) cause of decreasing optical performance of the element with temperature. These tests resulted in the redesign of the SST-1M composite mirrors. First of all symmetrization of the SMC structure and gel-coat was introduced, together with a reduction in the thickness of the gel-coat layer. Some further changes in the design which improve the technology of the mirror production are being implemented, and the SMC mirror facet technology is close to be finalized within next months. The second batch of SMC mirror prototypes will be tested on the SST-1M telescope structure developed by Institute of Nuclear Physics Polish Academy of Sciences in Krakow [21]. 
Acknowledgments The authors wish to thank Prof. Paula Chadwick from Durham University and Dr. Andreas Foerster from Max Planck Institute for Nuclear Physics for their helpful reviews and suggestions.

Financial support has been provided by Ministry of Science and Higher Education through grant 498/ FNiTP/158/2011 and statutory funds of the Warsaw University of Technology.

Open Access This article is distributed under the terms of the Creative Commons Attribution 4.0 International License (http://creativecommons.org/licenses/by/4.0/), which permits unrestricted use, distribution, and reproduction in any medium, provided you give appropriate credit to the original author(s) and the source, provide a link to the Creative Commons license, and indicate if changes were made.

\section{References}

1. Acharya, B.S., Actis, M., Aghajani, T., et al.: For the CTA Consortium, Introducing the CTA concept. Astropart. Phys. 43, 3-18 (2013)

2. Moderski, R., Aguilar, J.A., Barnacka, A., Płatos, Ł., Seweryn, K., et al.: For the CTA Consortium, 4 m Davies-Cotton telescope for the Cherenkov Telescope Array, arXiv:1307.3137 [astro-ph.IM] (2013)

3. Davies, J.M., Cotton, E.S.: Design of the quartermaster solar furnace. Sol. Energy 1(2-3), 16-22 (1957)

4. Boccone, V., Aguilar, J. A., Basili, A., Christov, A., della Volpe, M., Montaruli, T., Rameez, M.: For the CTA Consortium, The Photodetector Plane of the $4 \mathrm{~m}$ Davies Cotton Small Size Telescope for the Cherenkov Telescope Array, arXiv:1307.2723 [astro-ph.IM] (2013)

5. Pareschi, G., Armstrong, T., Baba, H., Bähr, J., et al.: for the CTA Consortium, Status of the technologies for the production of the Cherenkov Telescope Array mirrors. Proc. SPIE 8861, 886103 (2013)

6. Orgéas, L., Dumont, P. J. J.: Sheet Molding Compounds. Wiley Encyclopedia of Composites. 1-36 (2012)

7. Sutton, M., Orteu, J. J., Schreier, H.: Image Correlation for shape, motion and deformation measurements. Springer (2009)

8. Pan, B.: Recent progress in digital image correlation. Exp. Mech. 51(7), 1223-1235 (2011)

9. Hartley, R., Zisserman, A.: Multiple View Geometry in Computer Vision. Cambridge University Press, (2003)

10. Piekarczuk, A., Malesa, M., Kujawińska, M., Malowany, K.: Application of hybrid FEM-DIC method for assessment of low cost building structures. Exp. Mech. 52(9), 1297-1311 (2012)

11. Malesa, M., Malowany, K., Tomczak, U., Siwek, B., Kujawińska, M., Siemińska-Lewandowska, A.: Application of $3 \mathrm{~d}$ digital image correlation in 179 maintenance and process control in industry. Comput. Ind. 64, 1301-1315 (2013)

12. Jin, H., Bruck, H.A.: Pointwise digital image correlation using genetic algorithms. Exp. Tech. 29(1), 3639 (2005)

13. Zhang, Z.-F., Kang, Y.-L.: HWang.-W., Qin Q.-H., Qiu Y., Li X.-Q., A novel coarse-fine search scheme for digital image correlation method. Measurement 39(8), 710-718 (2006)

14. Pan, B.: Reliability-guided digital image correlation for image deformation measurement. Appl. Opt. 48(8), 1535-1542 (2009)

15. Hu, Z., Xie, H., Lu, J., Hua, T., Zhu, J.: Study of the performance of different subpixel image correlation methods in 3d digital image correlation. Appl. Opt. 49(21), 4044-4051 (2010)

16. Nguyen, T.N., Huntley, J.M., Burguete, R.L., Coggrave, C.R.: Shape and displacement measurement of discontinuous surfaces by combining fringe projection and digital image correlation. Opt. Eng. 50(10), 101505 (2011)

17. Pan, B.: Bias error reduction of digital image correlation using gaussian pre-filtering. Opt. Lasers Eng. 51, 1161-1167 (2013)

18. Pan, B., Li, K., Tong, W.: Fast, robust and accurate digital image correlation calculation without redundant computations. Exp. Mech. (2013). doi:10.1007/s11340-013-9717-6

19. Wang, Y.-Q., Sutton, M.A., Ke, X.-D., Schreier, H.W.: Error Propagation in Stereo Vision: Part I: Theoretical Developments. Exp. Mech. 51(4), 405-422 (2011)

20. Ke, X.-D., Schreier, H.W., Sutton, M.A., Wang, Y.-Q.: Error Propagation in Stereo Vision: Part II: Experimental Validation. Exp. Mech. 51(4), 423-441 (2011)

21. Niemiec, J., et al.: For the CTA Consortium, Single-Mirror Small-Size Telescope Structure for the Cherenkov Telescope Array, arXiv:1307.4189 [astro-ph.IM] (2013) 